\title{
Clima social familiar $y$ autoconcepto en adolescentes de una institución educativa de Lima Norte
}

\author{
Family social climate and self-concept in adolescents of an educational \\ institution in North Lima
}

\author{
Llanca-Sánchez, Billy Balvino ${ }^{1}$ \\ Armas-Zambrano, Nélida ${ }^{2}$
}

\begin{abstract}
Resumen
Objetivo: determinar la relación entre clima social familiar y autoconcepto en adolescentes de una Institución Educativa de Lima Norte. Materiales y métodos: el tipo de muestreo fue no probabilístico. La muestra estuvo conformada por 210 alumnos de ambos sexos, entre los 12 y 17 años de edad. Se usaron como instrumentos el Clima Social Familiar (FES), Cuestionario de Autoconcepto de Garley (CAG) y una ficha sociodemográfica. Para el análisis de los datos se utilizó el paquete estadístico Stata versión 14. Se empleó el Chi-cuadrado en el análisis de las variables categóricas. Se consideró una significancia estadística menor o igual a 0.05. Resultados: no se halló relación significativa entre Clima Social Familiar y Autoconcepto. EL $81.4 \%$ de los adolescentes se encontraron dentro del nivel muy mala en la dimensión relaciones del Clima Social Familiar y el $70.0 \%$ obtuvo un nivel de Autoconcepto medio. Por otra parte, se encontró relación significativa entre la dimensión estabilidad del clima social familiar y la dimensión familiar del autoconcepto $(\mathrm{p}=0.013)$. Conclusión: el clima social familiar y el autoconcepto no se relacionan significativamente; sin embargo, se hallaron algunas relaciones significativas entre algunas dimensiones de estas variables. Esto sugiere que en el hogar el desempeño de las responsabilidades de los padres de familia, podría relacionarse al autoconcepto de los adolescentes y a la forma en que estos se desempeñan en el entorno.
\end{abstract}

Palabras clave: Clima social familiar; Autoconcepto; Adolescentes (Fuente: DeCS).

\begin{abstract}
Objective: to determine the relationship between family social climate and self-concept in adolescents from an Educational Institution in Lima Norte. Materials and methods: the type of sampling was non-probabilistic. The sample was made up of 210 students of both sexes, between 12 and 17 years of age. The Family Social Climate (FES), the Garley Self-Concept Questionnaire (CAG) and a sociodemographic record were used as instruments. The Stata version 14 statistical package was used to analyze the data. Chi-square was used in the analysis of the categorical variables. Statistical significance less than or equal to 0.05 was considered. Results: no significant relationship was found between Family Social Climate and Self-concept. $81.4 \%$ of the adolescents were within the very poor level in the relations dimension of the Family Social Climate and $70.0 \%$ obtained a medium level of Self-concept. On the other hand, a significant relationship was found between the stability dimension of the family social climate and the family dimension of self-concept $(\mathrm{p}=0.013)$. Conclusion: family social climate and self-concept are not significantly related; however, some significant relationships were found between some dimensions of these variables. This suggests that the performance of parental responsibilities in the home could be related to the self-concept of adolescents and the way in which they perform in the environment.
\end{abstract}

Keys words: Family social climate; Self Concept; Teenagers (Source: DeCS).

Para citar:

Llanca BB, Arnas N. Clima social familiar y autoconcepto en adolescentes de una institución educativa de Lima Norte. CASUS. 2020;5(1):26-33.

DOI: $10.35626 /$ casus.1.2020.245

\footnotetext{
${ }^{1}$ Hospital Nacional Cayetano Heredia.

${ }^{2}$ Centro Educativo Parroquial San Vicente Ferrer.

Correo electrónico: billybalvino@gmail.com
}

Fecha de recepción: 11-01-20

Fecha de envío a pares: 14-01-20

Fecha de aprobación por pares: 12-03-20

Fecha de aceptación: 15-03-20 


\section{INTRODUCCIÓN}

El clima social familiar (CSF) comprende un conjunto de factores que caracterizan las interacciones entre los miembros de una familia. Estos factores psicológicos son los estilos de comunicación, la organización jerárquica familiar, el seguimiento de normas y códigos intra familiares (1). Asimismo, los lazos afectivos predominantes y las cogniciones que maneja la familia dentro del contexto psicosocial. En el CSF también influyen factores tangibles como la situación económica, política y cultural que rodea a la familia (2). El CSF está relacionado a una atmósfera psicológica donde se interactúan las características psicológicas de un grupo humano (familia) que se desenvuelve en diversos contextos, como pueden ser la escuela, el ámbito laboral, la comunidad, entre otros (1). Igualmente, tiene relación con diferentes variables psicológicas individuales como la manera en la que el sujeto se concibe y percibe, es decir, el autoconcepto y la autovaloración (2).

En este sentido, el autoconcepto es definido como la característica de una estructura psicológica en la que el sujeto interpreta la observación, el juicio y crítica de las demás personas (2). De esta manera, es un factor psicológico interno que determina considerablemente las cogniciones, el estado afectivo y comportamiento social del individuo (3). Cabe señalar que el CSF y el autoconcepto pueden estar relacionados ya que el entorno familiar está estrechamente vinculado a las percepciones del adolescente de sí mismo en otros contextos cercanos como la escuela (3).

De esta manera, resulta importante continuar estudiando la relación entre eL CSF y el autoconcepto en diversos contextos a nivel nacional. Anteriormente, estudios internacionales aportaron información indicando que los elementos del clima social familiar tienen importancia en el autoconcepto y en los niveles de adaptación personal de los adolescentes (4-13). Así mismo, otro estudio señala que un elevado autoconcepto general previene la aparición de comportamientos característicos de inadaptación personal como ansiedad, depresión, sentimiento de rechazo, estrés ante situaciones de la vida cotidiana e, incluso, del abuso de sustancias (14).
En el contexto nacional, se ha destacado que el CSF se vincula con el autoconcepto. Es decir, los adolescentes cuyas familias han desarrollado una atmósfera agradable y favorable para interactuar manifiestan simultáneamente una adecuada evaluación de sí mismos (15). Igualmente, se realizó un estudio sobre esta relación en adolescentes de la ciudad de Huancayo (Perú) hallándose que los mismos presentaron un alto autoconcepto vinculado a la libre expresión de sus ideas y emociones en un contexto familiar funcional (16).

No obstante, es necesario continuar profundizando en las características de relación entre ambos constructos debido a que no se a explorado suficientemente en contextos de bajos ingresos o se ha pasado por alto elementos de la estructura familiar de los adolescentes. Estas variables pudieran ayudar a dar más datos contextuales para la comprensión de esta asociación. Considerando estos vacíos de conocimiento, la situación planteada y los antecedentes mencionados, el estudio tuvo por objetivo identificar la relación existente entre clima social familiar $y$ autoconcepto en adolescentes de una institución educativa del distrito de Comas en Lima Norte.

\section{MATERIALES Y MÉTODOS}

Estudio correlacional de corte transversal. La población estuvo conformada por 460 adolescentes del primero al quinto de secundaria de una institución educativa de Lima Norte. El muestreo fue no probabilístico intencional. La muestra estuvo compuesta por 210 adolescentes, de ambos sexos.

Las variables principales fueron el clima social familiar (variable categórica politómica ordinal) y el autoconcepto (variable categórica politómica ordinal). Las variables sociodemográficas fueron: edad (variable cuantitativa discreta), sexo (variable cualitativa dicotómica), distrito de proveniencia (variable cualitativa politómica nominal) y tipo de familia (variable categórica politómica nominal) recolectadas con una ficha de datos.

Para la recolección de información se utilizó la Escala de Clima Social Familiar (FES) $(1,16)$ que 
Tabla 1. Descripción de las variables principales y los datos sociodemográficos

\begin{tabular}{|c|c|c|}
\hline & $\mathbf{n}$ & $\%$ \\
\hline Edad (media/DE) & \multicolumn{2}{|c|}{$13.56 \pm 1.40$} \\
\hline \multicolumn{3}{|l|}{ Sexo } \\
\hline Femenino & 94 & 44.8 \\
\hline Masculino & 116 & 55.2 \\
\hline \multicolumn{3}{|l|}{ Tipo de familia } \\
\hline Monoparental & 22 & 10.5 \\
\hline Nuclear & 119 & 56.7 \\
\hline Extensa & 69 & 32.9 \\
\hline \multicolumn{3}{|l|}{ Distrito } \\
\hline Comas & 208 & 99.1 \\
\hline Carabayllo & 1 & 0.5 \\
\hline Ventanilla & 1 & 0.5 \\
\hline \multicolumn{3}{|c|}{ Clima Social Familiar } \\
\hline Promedio & 17 & 8.1 \\
\hline Mala & 147 & 70.0 \\
\hline Muy mala & 46 & 21.9 \\
\hline \multicolumn{3}{|l|}{ Relaciones } \\
\hline Excelente & 1 & 0.5 \\
\hline Promedio & 5 & 2.4 \\
\hline Mala & 33 & 15.7 \\
\hline Muy mala & 171 & 81.4 \\
\hline \multicolumn{3}{|l|}{ Desarrollo } \\
\hline Promedio & 2 & 0.9 \\
\hline Mala & 43 & 20.5 \\
\hline Muy mala & 165 & 78.6 \\
\hline \multicolumn{3}{|l|}{ Estabilidad } \\
\hline Promedio & 31 & 14.8 \\
\hline Mala & 123 & 58.6 \\
\hline Muy mala & 56 & 26.7 \\
\hline \multicolumn{3}{|l|}{ Autoconcepto } \\
\hline Alto & 23 & 10.9 \\
\hline Medio & 147 & 70.0 \\
\hline Bajo & 40 & 19.1 \\
\hline \multicolumn{3}{|l|}{ Físico } \\
\hline Alto & 59 & 28.1 \\
\hline Medio & 80 & 38.1 \\
\hline Bajo & 71 & 33.8 \\
\hline \multicolumn{3}{|l|}{ Social } \\
\hline Alto & 57 & 27.1 \\
\hline Medio & 87 & 41.4 \\
\hline Bajo & 66 & 31.4 \\
\hline \multicolumn{3}{|l|}{ Familiar } \\
\hline Alto & 5 & 2.4 \\
\hline Medio & 85 & 40.5 \\
\hline Bajo & 120 & 57.1 \\
\hline \multicolumn{3}{|l|}{ Intelectual } \\
\hline Alto & 31 & 14.8 \\
\hline Medio & 83 & 39.5 \\
\hline Bajo & 96 & 45.7 \\
\hline \multicolumn{3}{|l|}{ Personal } \\
\hline Alto & 67 & 31.9 \\
\hline Medio & 79 & 37.6 \\
\hline Bajo & 64 & 30.5 \\
\hline
\end{tabular}

Tabla 1. continúa

\begin{tabular}{ccc}
\hline & n & \% \\
\hline Control & & \\
Alto & 11 & 5.2 \\
Medio & 92 & 43.8 \\
Bajo & 107 & 50.9 \\
\hline
\end{tabular}

consta de 90 ítems y comprende tres dimensiones: relación, desarrollo y estabilidad. Los puntajes de las dimensiones se categorizaron. Dimensión relación: muy buena (21 a más), buena (20), tendencia buena (19), media (14 a 18), mala (12 a 13) y muy mala (0 a 11). Dimensión desarrollo: muy buena (33 a más), buena (32), tendencia buena (30 -31), media (25 a 29), mala (19 a 24) y muy mala (0 a 18). Dimensión estabilidad: muy buena (18 a más), buena (17), tendencia buena (16), media (11 a 15), mala (8 a 10) y muy mala (0 a 7).

También se empleó el Cuestionario de Autoconcepto de Garley (CAG) con 48 ítems y abarcando 6 dimensiones. Los puntajes de las dimensiones fueron categorizados, dimensión física: alto (35 a 40), medio (28 a 34), bajo (11 a 27). Dimensión social: alto (33 a 40), medio (26 a 32), bajo (10 a 25). Dimensión familiar: alto (36 a 40), medio (30 a 35), bajo (11 a 29). Dimensión intelectual: alto (34 a 40), medio (27 a 33), bajo (14 a 26). Dimensión personal: alto (36 a 40), medio (31 a 35), bajo (16 a 30). Dimensión control: alto (34 a 40), medio (27 a 33), bajo (16 a 26) (2).

Para el análisis de los datos se utilizó el paquete estadístico Stata versión 14. También se empleó la prueba estadística Chi-cuadrado en el análisis de las variables categóricas. Se consideró una significancia estadística menor o igual a 0.05 . El presente estudio fue aprobado por el Comité de Ética de la Universidad Católica Sedes Sapientiae y los datos de los participantes fueron protegidos de acuerdo a principios bioéticos.

\section{RESULTADOS}

De los 210 estudiante evaluados se pudo apreciar que la edad promedio fue de 13.56 y el $55.2 \%$ de sexo masculino. El $56.7 \%$ reportó un tipo de 
Tabla 2. Relación entre el clima social familiar, el autoconcepto y los datos sociodemográficos

\begin{tabular}{|c|c|c|c|c|}
\hline & $\begin{array}{c}\text { Promedio } \\
\mathrm{n}(\%)\end{array}$ & $\begin{array}{l}\text { Mala } \\
\mathrm{n}(\%)\end{array}$ & $\begin{array}{c}\text { Muy mala } \\
\text { n (\%) }\end{array}$ & p-valor \\
\hline $\begin{array}{l}\text { Edad } \\
\text { (media/DE) }\end{array}$ & $\begin{array}{c}12.82 \pm \\
1.01\end{array}$ & $\begin{array}{c}13.57 \pm \\
1.40\end{array}$ & $\begin{array}{c}13.78 \pm \\
1.47\end{array}$ & 0.055 \\
\hline Sexo & & & & 0.085 \\
\hline Femenino & $8(8.5)$ & $59(62.8)$ & 27 (28.7) & \\
\hline Masculino & $9(7.8)$ & 88 (75.9) & $19(16.4)$ & \\
\hline Tipo de familia & & & & 0.986 \\
\hline Monoparental & $2(9.1)$ & $16(72.7)$ & $4(18.1)$ & \\
\hline Nuclear & $9(7.6)$ & $84(70.6)$ & $26(21.9)$ & \\
\hline Extensa & $6(8.7)$ & $47(68.1)$ & $16(23.2)$ & \\
\hline Distrito & & & & 0.406 \\
\hline Comas & $17(8.2)$ & $146(70.2)$ & 45 (21.6) & \\
\hline Carabayllo & $0(0)$ & $0(0)$ & $1(100)$ & \\
\hline Ventanilla & $0(0)$ & $1(100)$ & $0(0)$ & \\
\hline Autoconcepto & & & & 0.874 \\
\hline Alto & $2(8.7)$ & $14(60.9)$ & $7(30.4)$ & \\
\hline Medio & $12(8.2)$ & $104(70.8)$ & $31(21.1)$ & \\
\hline Bajo & $3(7.5)$ & $29(72.5)$ & $8(20.0)$ & \\
\hline Físico & & & & 0.768 \\
\hline Alto & $6(10.2)$ & $40(67.8)$ & $13(22.0)$ & \\
\hline Medio & $4(5.0)$ & $59(73.8)$ & $17(21.3)$ & \\
\hline Bajo & $7(9.9)$ & $48(67.6)$ & $16(22.5)$ & \\
\hline Social & & & & 0.098 \\
\hline Alto & $3(5.3)$ & 37 (64.9) & $17(29.8)$ & \\
\hline Medio & $11(12.6)$ & $63(72.4)$ & 13 (14.9) & \\
\hline Bajo & $3(4.6)$ & $47(71.2)$ & $16(24.2)$ & \\
\hline Familiar & & & & 0.225 \\
\hline Alto & $0(0)$ & $2(40.0)$ & $3(60.0)$ & \\
\hline Medio & $7(8.2)$ & $57(67.1)$ & $21(24.7)$ & \\
\hline Bajo & $10(8.3)$ & $88(73.3)$ & $22(18.3)$ & \\
\hline Intelectual & & & & 0.399 \\
\hline Alto & $3(9.7)$ & $23(74.2)$ & $5(16.1)$ & \\
\hline Medio & $7(8.4)$ & $62(74.7)$ & $14(16.9)$ & \\
\hline Bajo & $7(7.3)$ & $62(64.6)$ & $27(28.1)$ & \\
\hline Personal & & & & 0.371 \\
\hline Alto & $7(10.5)$ & $41(61.2)$ & $19(28.4)$ & \\
\hline Medio & $6(7.6)$ & $60(75.9)$ & $13(16.5)$ & \\
\hline Bajo & $4(6.3)$ & 46 (71.9) & 14 (21.9) & \\
\hline Control & & & & 0.991 \\
\hline Alto & $1(9.1)$ & $8(72.7)$ & $2(18.2)$ & \\
\hline Medio & $8(8.7)$ & $63(68.5)$ & $21(22.8)$ & \\
\hline Bajo & $8(7.5)$ & $76(71.0)$ & $23(21.5)$ & \\
\hline
\end{tabular}

familia nuclear. En relación al CSF del 70\% de los participantes fue clasificado como mala. Se encontró que las dimensiones relaciones $(81.4 \%)$ y desarrollo $(71.6 \%)$ fueron clasificadas en un nivel de muy mala. Mientras que, en la dimensión estabilidad en un nivel de mala (58.6\%). Por otro lado, se halló que el $70 \%$ presentó un nivel medio de autoconcepto en general. No obstante, se evidenció que en las dimensiones físico (38.1\%),
Tabla 3. Relación entre el autoconcepto y los datos sociodemográficos

\begin{tabular}{lcccc}
\hline & \multicolumn{4}{c}{ Autoconcepto } \\
& $\begin{array}{c}\text { Alto } \\
\text { Medio }\end{array}$ & $\begin{array}{c}\text { Bajo } \\
\text { n }(\%)\end{array}$ & $\begin{array}{c}\text { p- } \\
\text { valor }\end{array}$ \\
\hline Edad & $13.56 \pm$ & $13.48 \pm$ & $13.75 \pm$ & 0.467 \\
(media/DE) & 1.42 & 1.38 & 1.50 & \\
Sexo & & & & 0.760 \\
$\quad$ Femenino & $10(10.6)$ & $64(68.1)$ & $20(21.3)$ & \\
$\quad$ Masculino & $13(11.2)$ & $83(71.6)$ & $20(17.2)$ & \\
Tipo de familia & & & & 0.813 \\
$\quad \begin{array}{l}\text { Monoparental } \\
\text { Nuclear }\end{array}$ & $4(18.2)$ & $14(63.6)$ & $4(18.2)$ & \\
$\quad$ Extensa & $6(8.7)$ & $50(72.5)$ & $13(18.8)$ & \\
Distrito & & & & 0.320 \\
$\quad$ Comas & $23(11.1)$ & $146(70.2)$ & $39(18.8)$ & \\
$\quad \begin{array}{l}\text { Carabayllo } \\
\text { Ventanilla }\end{array}$ & $0(0)$ & $1(100.0)$ & $0(0)$ & \\
\hline
\end{tabular}

social $(41.4 \%)$ y personal $(37.6 \%)$ presentaron un nivel medio, y en las dimensiones familiar (57.1\%), intelectual (45.7\%) y control (50.9\%) presentaron un nivel bajo. El resto de los datos se observa en la tabla 1.

No se encontró relación significativa entre el CSF, el autoconcepto y los datos sociodemográficos (ver tabla 2). Igualmente, no se encontró relación significativa entre el autoconcepto y los datos sociodemográficos (ver tabla 3). Se evidenció, por otra parte, relación significativa entre la dimensión estabilidad del clima social familiar con la dimensión familiar del autoconcepto $(\mathrm{p}=0.013)$ (ver tabla 4).

\section{DISCUSIÓN}

En la muestra del estudio se halló que no existe relación estadística significativa entre el clima social familiar y el autoconcepto. Sin embargo, existe relación significativa entre la dimensión estabilidad del clima social familiar y la dimensión familiar del autoconcepto. Además, se apreció que existe una mayoría de adolescentes que clasifica para los niveles mala y muy mala del CSF. También se halló que el nivel del autoconcepto predominante fue el medio. Se observó que en las dimensiones familiar, intelectual y control del autoconcepto los adolescentes se ubicaron en el nivel bajo. 
Tabla 4. Relación entre la dimensión estabilidad del clima social familiar, el autoconcepto y los datos sociodemográficos

\begin{tabular}{|c|c|c|c|c|}
\hline & \multicolumn{4}{|c|}{ Estabilidad } \\
\hline & $\begin{array}{c}\text { Promedio } \\
\mathrm{n}(\%)\end{array}$ & $\begin{array}{l}\text { Mala } \\
\mathrm{n}(\%)\end{array}$ & $\begin{array}{c}\text { Muy mala } \\
\mathrm{n}(\%)\end{array}$ & $\mathrm{p}$-valor \\
\hline Edad (media/DE) & $13.58 \pm 1.31$ & $13.63 \pm 1.40$ & $13.39 \pm 1.46$ & 0.497 \\
\hline Sexo & & & & 0.816 \\
\hline Femenino & $13(13.89$ & $54(57.5)$ & $27(28.7)$ & \\
\hline Masculino & $18(15.5)$ & $69(59.5)$ & $29(25.0)$ & \\
\hline Tipo de familia & & & & 0.944 \\
\hline Monoparental & $2(9.1)$ & $14(63.6)$ & $6(27.3)$ & \\
\hline Nuclear & $19(15.9)$ & $68(57.1)$ & $32(26.9)$ & \\
\hline Extensa & $10(14.5)$ & $41(59.4)$ & $18(26.1)$ & \\
\hline Distrito & & & & 0.073 \\
\hline Comas & $30(14.4)$ & $123(59.1)$ & $55(26.4)$ & \\
\hline Carabayllo & $0(0)$ & $0(0)$ & $1(100.0)$ & \\
\hline Ventanilla & $1(100.0)$ & $0(0)$ & $0(0)$ & \\
\hline Autoconcepto & & & & 0.645 \\
\hline Alto & $2(8.7)$ & $12(52.2)$ & $9(39.1)$ & \\
\hline Medio & $22(14.9)$ & $88(59.9)$ & $37(25.2)$ & \\
\hline Bajo & $7(17.5)$ & $23(57.5)$ & $10(25.0)$ & \\
\hline Físico & & & & 0.548 \\
\hline Alto & 7 (11.9) & $34(57.6)$ & $18(30.5)$ & \\
\hline Medio & $10(12.5)$ & $51(63.8)$ & $19(23.8)$ & \\
\hline Bajo & $14(19.7)$ & $38(53.5)$ & $19(26.8)$ & \\
\hline Social & & & & 0.253 \\
\hline Alto & $7(12.3)$ & $32(56.1)$ & $18(31.6)$ & \\
\hline Medio & $18(20.7)$ & $50(57.5)$ & $19(21.8)$ & \\
\hline Bajo & $6(9.1)$ & $41(62.1)$ & $19(28.8)$ & \\
\hline Familiar & & & & 0.013 \\
\hline Alto & $1(20.0)$ & $0(0)$ & $4(80.0)$ & \\
\hline Medio & $8(9.4)$ & $51(60.0)$ & $26(30.6)$ & \\
\hline Bajo & $22(18.3)$ & $72(60.0)$ & $26(21.7)$ & \\
\hline Intelectual & & & & 0.331 \\
\hline Alto & $5(16.1)$ & $19(61.3)$ & $7(22.5)$ & \\
\hline Medio & $10(12.1)$ & $55(66.3)$ & $18(21.7)$ & \\
\hline Bajo & $16(16.7)$ & $49(51.0)$ & $31(32.3)$ & \\
\hline Personal & & & & 0.199 \\
\hline Alto & $9(13.4)$ & $38(56.7)$ & $20(29.9)$ & \\
\hline Medio & $8(10.1)$ & $53(67.1)$ & $18(22.8)$ & \\
\hline Bajo & $14(21.9)$ & $32(50.0)$ & $18(28.1)$ & \\
\hline Control & & & & 0.966 \\
\hline Alto & $1(9.1)$ & 7 (63.6) & $3(27.3)$ & \\
\hline Medio & $14(15.2)$ & $52(56.5)$ & $26(28.3)$ & \\
\hline Bajo & $16(14.9)$ & $64(59.8)$ & $27(25.2)$ & \\
\hline
\end{tabular}

En el estudio no se encontró relación significativa entre el CSF y el autoconcepto. Sin embargo, en la literatura algunos autores hallaron relación significativa entre estas variables (15-19). En el presente trabajo la ausencia de relación significativa podría deberse a factores socioeconómicos, socioculturales y psicológicos de los adolescentes y sus familiares. Provenir de familias del interior del país en las cuales los progenitores exigen a los adolescentes lograr un desempeño adecuado en base normas y reglas, sin permitir expresar sus ideas, emociones y necesidades (20). De esta manera el adolescente no recibe motivación de su entorno para lograr alcanzar sus logros dentro de la institución educativa. Por ello, pudiera manifestar dificultades en sus habilidades sociales y temor a adaptarse ante diferentes situaciones dentro la escuela.

Sin embargo, se halló relación significativa entre la dimensión estabilidad del CSF y la dimensión familiar del autoconcepto. En otro estudio se 
confirmó esta relación indicando que un elevado porcentaje de estudiantes con una buena estabilidad familiar presentan una autoestima y autoconcepto adecuados (21). El mayor porcentaje de tipo de familia reportado fue nuclear lo cual podría indicar que el hogar de los adolescentes está estructurado y organizado. Lo anterior se relaciona al grado de control de los miembros. Además, la relación conjuntamente al grado de satisfacción y la aceptación familiar les proporciona una visión positiva de sí mismos.

Los adolescentes en su mayoría presentan los niveles del clima social malo y muy malo. Así mismo, se evidenció que el nivel malo del CSF presenta mayor porcentaje en sus tres dimensiones. Este dato pudiera sugerir que existe en los hogares una mala comunicación, mala relación en los miembros del hogar, un ambiente desfavorable sin una organización y reglas que no permiten una adecuada expresión de sus emociones (22). Estos niveles pudieran estar asociados a la presencia de disfuncionalidad familiar. Esta se caracteriza por la limitación en la autonomía de los miembros, generando inseguridad. Asimismo, en los entornos familiares de los sujetos de la muestra se podrían presentar escasas relaciones interpersonales positivas, trato empático, una convivencia cordial, poca voluntad de servicio entre sus miembros. En esta línea, se halló en la ciudad de Lima una alta prevalencia de divorcios rápidos en las municipalidades, maltrato físico y psicológico dentro del hogar y problemas de conducta en los adolescentes vinculados a la disfuncionalidad familiar (23).

Por otra parte, se halló que el nivel de autoconcepto predominante fue el medio. Otro estudio hace referencia que los niveles de autoconcepto entre los adolescentes son de nivel medio (24). Además, este elemento señala que, posiblemente, el autoconcepto dependa del juicio de las demás personas y de cómo nos vemos a nosotros mismos (24).

También se halló que las dimensiones familiar, intelectual y control del autoconcepto de los adolescentes se ubicaron en el nivel bajo. Un estudio reportó igualmente que la dimensión familiar y control presentaban un nivel bajo (16). Este elemento pudiera indicar que el adolescente presentaría una inadecuada relación dentro de su hogar lo que puede generar inseguridad para identificarse con sus figuras paternales. Por el contrario, en la dimensión intelectual presentaron un nivel alto lo cual hace presumir que los adolescentes se sienten seguros de sus capacidades antes los exámenes, trabajos o tareas que desempeña fuera y dentro del colegio logrando esto por su organización y la supervisión dentro de su familia (16).

Entre las limitaciones del estudio puede señalarse que, al tener un alcance correlacional, no es posible establecer relaciones de causa-efecto entre las variables del estudio. Asimismo, al ser un estudio transversal, no fue posible conocer el comportamiento de las variables en el tiempo. Añadido a lo anterior, las pruebas de autoaplicación pudieron estar sujetas a deseabilidad social entre los participantes. Sin embargo, el tamaño muestral es representativo y los resultados generalizables a poblaciones adolescentes de instituciones educativas similares.

\section{CONCLUSIONES}

Aun cuando el clima social familiar y el autoconcepto no se relacionaron estadísticamente, se hallaron algunas significancias entre algunas dimensiones de estas variables. Esto sugiere que en el hogar la manera en que los padres desempeñan sus responsabilidades podría relacionarse al autoconcepto de los adolescentes y a la forma en que estos perciben y actúan en el entorno. Lo anterior podría estar conectado a diferencias en la capacidad de adaptabilidad de los adolescentes frente a las demandas del contexto.

La relación significativa entre la dimensión estabilidad del clima social familiar y la dimensión familiar del autoconcepto podría evidenciar que dentro del hogar la estructura y desempeño de las responsabilidades pueden relacionarse al autoconcepto y a la manera en que el adolescente se relaciona con su familia. 
En la muestra de estudio predominaron el clima social familiar malo y el nivel bajo de autoconcepto. Estos indicadores, en conjunto, podrían resultar en cogniciones personales y afectos negativos acerca de sí mismo y su

\section{REFERENCIAS BIBLIOGRÁFICAS}

1. Moos R. y Trickett. E. Escala de Clima Social en la Familia (FES). Estados Unidos:1993.

2. García B. Cuestionario del 8. Vera J, Morales D, Vera C. autoconcepto Garley (CAG). España: 2001.

3. Malca A, Rivera L. Clima social familiar. ¿Qué relación tiene con el autoconcepto en adolescentes del Callao? CASUS. 2019;4(2):120-129.

4. Álvarez L. Clima Social Familiar en estudiantes de bachillerato víctimas de acoso escolar de una institución educativa. [Tesis de licenciatura]. Ecuador: Universidad de Cuenca; 2019.

5. Reyes V, Merino T, Villavicencio L. Mendoza C, Sequera A. El clima social familiar y la agresividad en educación primaria: un estudio de caso en Guayaquil Ecuador. Research, Society and Development. 2019; 8(11):25253409 .

6. Vargas J. Percepción del clima social familiar y actitudes ante situaciones de agravio en la adolescencia tardía. Interdisciplinaria Revista de Psicología y Ciencias Afines. 2009; 26(2):289-316.

7. Moreno D, Estévez E, Murgui S, Musitu G. Relación entre el clima social familiar y clima escolar: el rol de la empatía, la actitud hacia la autoridad y la conducta violenta en la adolescencia. International autoaceptación, así como comportamientos no productivos a nivel familiar y en los diferentes entornos que frecuenta el adolescente.
Journal of Psychology and 13. Gómez L. Clima escolar social y Psychological Therapy. 2009; 9(1):123-136.

Relaciones del desarrollo cognitivo con el clima familiar y el estrés de la crianza. Revista Psico USF. 2005; 10(2):161-168.

Moscol C. Relación entre el clima social familiar y autoestima en los estudiantes de tercer grado de nivel secundario turno tarde de la I. E. José Cayetano Heredia, Catacaos, Piura. [Tesis de 15. Guerrero, S \& Mestanza, N. Clima licenciatura]. Piura: Universidad Católica Los Ángeles de Chimbote; 2019

10. Díaz R, González D. Clima social familiar y conductas antisociales en adolescentes de una Institución Educativa Estatal - Cajamarca. [Tesis de licenciatura]. Cajamarca: La Universidad de Cajamarca; 2019.

11. Benavides D, Calle A. Clima social familiar $\mathrm{y}$ habilidades sociales en estudiantes del nivel secundario de la Institución Educativa 0004 Túpac Amaru. [Tesis de licenciatura]. Tarapoto: Universidad Peruana Unión; 2019.

12. Baquerizo B, Geraldo E. Autoconcepto y habilidades 18. Antón, S. Relación entre el Clima sociales en los estudiantes del nivel secundario de la Institución Educativa Puerto Pizana, 2016. [Tesis de licenciatura]. Tarapoto: Universidad Peruana Unión; 2016. autoconcepto en alumnos de educación secundaria de Lima. [Tesis de licenciatura]. Lima: Universidad Nacional Mayor de San $\quad$ Marcos; 2010

14. Pichardo C, Fernández E, Amezcua J. Importancia del clima social familiar en la adaptación personal y social de los adolescentes. Revista de psicología general y aplicada. 2002;55(4):575-590.

Social Familiar y Autoconcepto de 3ro a 5to año de secundaria de la Institución Educativa de Lima Este, 2015. [Tesis de Licenciatura]. Lima: Universidad Peruana Unión; 2016

16. Yaranga, E. Clima social familiar y autoconcepto en estudiantes del cuarto grado de educación secundaria de la Institución Educativa Maestro RedentorHuancayo. [Tesis de Licenciatura]. Lima: Universidad Inca Garcilaso de la Vega; 2019.

17. Ruiz C, Guevara E. Estandarización de la Escala de Clima Social en la Familia (FES). Lima.

1993. social familiar y autoconcepto de los estudiantes de segundo y tercero de secundaria de la I.E José Gálvez Egusquiza Talandracas Provincia de Chulucanas Piura 2016 [Tesis de 
licenciatura]. Piura: Universidad Católica Los Ángeles Chimbote; 2018 .

19. Romero, C. El clima socia familiar y el autoconcepto en los alumnos del 3ero de secundaria de la institución educativa San Juan de San Juan de Miraflores. [Tesis de maestría]. Lima: Universidad César $\quad 2015$

20. Revuelta L, Esnaola I. Clima familiar deportivo y autoconcepto físico en la adolescencia. European Journal of Education and Psychology. 2011; 4(1): 19- Ángeles de Chimbote; 2016. 31.

23. Quiñe, M. Clima social familiar en estudiantes del tercer grado de educación secundaria de la Institución Pública Nro. 7008 Scripion Llona del distrito de Miraflores. [Tesis de Licenciatura]. Universidad Inca Garcilaso de la Vega. Lima; 2019. estabilidad del clima socia familiar de los estudiantes del tercer grado de Educación 24. Madrigales, C. Autoconcepto en Secundaria con buena autoestima en la Institución Educativa Francisco. [Tesis de Licenciatura]. Piura: Universidad Católica los adolescentes entre 14 y 18 años. [Tesis de Licenciatura] Guatemala: Universidad Rafael Landívar; 2012. 